\title{
The Compact Wheelchair Roller Dynamometer
}

\section{(ㄷ)(우)(}

\author{
Authors \\ Saulo Fernandes Melo Oliveira1, Afonso Augusto Guimarães Bione², Lúcia Inês Guedes Leite Oliveira ${ }^{3}$, Adalberto \\ Veronese da Costa ${ }^{4}$, Fernando José de Sá Pereira Guimarães ${ }^{5}$, Manoel da Cunha Costa ${ }^{5}$
}

\section{Affiliations}

1 Pernambuco State University, Human Performance Laboratory, Recife-PE, Brazil

2 Federal Rural University of Pernambuco, Distance Education Center, Recife, Brazil

3 Pernambuco State University, Associate Program of Post-Graduation in Physical Education UPE/UFPB, Recife, Brazil

4 University of the State of Rio Grande do Norte, Physical Education College, Mossoró, Brazil

5 UPE, Physical Education, Recife, Brazil

\section{Key words}

people with disabilities, locomotion, effort test, therapeutic exercise

received 15.12 .2016

revised 20.04.2017

accepted 05.05.2017

\section{Bibliography}

DOI https://doi.org/10.1055/s-0043-111404

Sports Medicine International Open 2017; 1: E119-E127

(c) Georg Thieme Verlag KG Stuttgart - New York

ISSN 2367-1890

\author{
Correspondence \\ MsC. Saulo Fernandes Melo Oliveira \\ Pernambuco State University, Human Performance \\ Laboratory \\ ESEF/UPE \\ Rua Arnóbio Marques, 310, Santo Amaro \\ Recife. PE. Brazil \\ CEP: $50.100-130$ \\ Tel.: + 55/84/3315-2131 \\ saulofmoliveira@gmail.com
}

\section{ABSTRACT}

The equipment for evaluating the propulsion of a wheelchair is very complex and expensive. To validate a new dynamometer prototype for assessing the propulsion capacity of wheelchairs, 21 healthy subjects (age: $20.9 \pm 2.4 \mathrm{yr}$; weight: $68.9 \pm 7.9 \mathrm{~kg}$; height: $174.0 \pm 7.1 \mathrm{~cm}$; BMI: $22.7 \pm 2.5 \mathrm{~kg} \cdot \mathrm{m}^{-2}$ ) who do not normally require wheelchairs performed a sprint protocol for $20 \mathrm{~s}$ after a 1-min warm-up. The power and rotation data acquired by the prototype (both right and left sides) were compared with those of a reference system via high-speed videography ( $240 \mathrm{fps}$ ). The results showed high levels of accordance $(95 \% \mathrm{Cl})$, excellent values for the intraclass correlation coefficient (ICC: .99; $P<0.00)$, no significant differences in the rotation $(P=0.91)$ and power $(P=0.94)$ between the methods. The proposed equipment met the validation criteria and thus can be applied as a new tool for assessing wheelchair propulsion.

\section{Introduction}

Most people with disabilities use wheelchairs that need to be propelled manually for activities of daily life and to participate in sports. The need to use their upper limbs instead of their lower limbs for activities is a paradigm shift in the lives of people with disabilities. This shift brings about several adaptations, including physiological [33], biomechanical [10, 19], and motor control [28] changes. This causes wheelchair users to constantly require evaluation in rehabilitation [3] (Collinger et al., 2008) and physical conditioning training $[18,32]$ in order to bear the workloads imposed on joints $[11,24]$ and muscles that are directly or indirectly involved in propulsion. For this type of demand in rehabilitation, it is necessary to have specific equipment for the evaluation of kinetic and kinematic qualities of manual wheelchair propulsion.
Electronic resources [2] and cylinder systems [30] are commonly used with video analysis to determine the dynamic characteristics of propulsion.

Few rehabilitation centers have equipment that can complement the information collected by electronic sensors (e. g., smart wheels) for simulating the wheelchair propulsion and rolling resistance under controlled conditions. This type of equipment is known as an inertial dynamometer [30]. It provides oversized models, complex calibration [5], and a braking load increase feature. It operates similarly to ergometric equipment and can influence the handling form and ergonomic contribution to the propulsive movement.

Equipment with mechanical or electromagnetic braking mechanisms imposes a greater energy demand on subjects than the existing resistance on the rolling course imposed by the equipment 
itself. Ergometers with these characteristics have been validated by other researchers [6]. Exerting a load that opposes the movement has the main objective of developing a cardiovascular and metabolic overload for individuals who use wheelchairs for physical training and sports [29].

However, people suffering from certain types of severe disabilities such as dystrophies, cerebral palsy, and quadriplegia may not be able to perform maximal or even submaximal effort protocols $[15,23]$. This equipment with load imposition characteristics is too costly for purchase in less developed countries or regions, which have significant numbers of people with disabilities who use manual wheelchairs. Thus, this group of people is assisted by public health services, which do not have specific instruments for this type of evaluation.

Recently, open-source development platforms have been developed to minimize the costs of programming and automation development. In the rehabilitation field, some tools have been produced and are increasingly being used in medical centers [16, 17]. Examples of these tools include the Arduino ${ }^{\circledR}$ (available at www. arduino.cc) and Raspberry pi ${ }^{\circledR}$ (available at raspberrypibra.com) platforms, which have the technology for numerous applications involving sensor interaction and the interpretation and presentation of data arising from the physical world or environment.

Based on the need for cost reduction, easier data acquisition, and the possibility of evaluating kinetic aspects (muscle strength and power) in people with different disabilities who use wheelchairs, the objectives of this research were as follows: a) to propose a new low-cost and compact method for the dynamometric evaluation of wheelchair users and $b$ ) to validate the acquisition system as well as the sampling of the rotation and power data.

\section{Methods}

\section{Ethical Research and Presumptions}

All research procedures were properly documented, and the research was registered with the Ethics Committee for Research on Humans of the University of Pernambuco (protocol no. 078/2011). All specified procedures followed national law. All research was conducted within the framework of the Declaration of Helsinki and has been performed in accordance with the ethical standards proposed by Harriss and Atkinson (2016) [37].

\section{Development of Prototype}

\section{Mechanical parts}

The compact dynamometer (i. e., DYNACOM) is a mechatronic system developed by researchers at the School of Physical Education in partnership with the Center for Remote Education, both of which are from the University of Pernambuco/Brazil. The main objective of this system is to evaluate a wheelchair's propulsive power, which allows it to be used as an instrument for locomotion and sports training. To accommodate the total weight of the subject and wheelchair, 2 systems of cylinders (Easy Scroll, Campinas, Brazil) were arranged in parallel and joined by lateral support rods. Each system comprised 3 cylinders separated by 2 distinct distances $(0.1$ and $0.2 \mathrm{~m})$ so that higher $(0.2 \mathrm{~m})$ and lower $(0.1 \mathrm{~m})$ resistances could be produced on the bearing during use. The wheelchair should be placed between 2 cylinders for testing, which may happen at a higher or lower rolling resistance. The resistance to the bearing constituted the force opposing the rotating motion of the wheels on a given surface [20], which increased with the inter-cylinder distance $(0.2 \mathrm{~m})$. The central cylinder was considered to be the instrumentation cylinder (CIN), where 2 metal circular objects were connected to drive the rotation sensors. Each cylinder had a length of $0.45 \mathrm{~m}$, circumference of $0.24 \mathrm{~m}$, and mass of $1.6 \mathrm{~kg}$.

\section{Electro-electronic parts}

The rotation data for the cylinders were collected by using 2 inductive-type rotation sensors (Sensorbrás, São Paulo, Brazil). For the transmission and interpretation of the electronic signs, we used the Arduino ${ }^{\circledR}$ open-source hardware and software (Arduino Mega, Atmega 2586, Italy), which were specially programmed to be a physical interface that makes use of information from various sources. The board was properly programmed to perform measurements at intervals of less than $0.001 \mathrm{~s}$. These readings were simultaneously transmitted to a personal computer for sampling and storage in XLSX or XLS files (MS Office 2007, Excel for Windows). The communication between the Arduino ${ }^{\circledR}$ board and Excel was realized with the PLX-DAQ software (Parallax, United States).

- Table 1 (Appendix) provides a complete description of this system, including all parts and the equipment used for the prototype assembly, along with the technical specifications and their actual values. Note that the parts were purchased at their average prices in Brazil when the equipment was assembled. The machine had a total estimated cost of approximately USD 450.00, did not weigh more than $40 \mathrm{~kg}$, and could be transported to training sites for the rehabilitation of wheelchair users.

Determination of propulsive power

To determine the propulsive power, the following relation between the load and rotational movement was used:

$P O(W)=\frac{F \cdot d \cdot n}{t}$

where $\mathrm{W}$ is the power (in watts), $\mathrm{F}$ ( $\mathrm{kg}$ or $\mathrm{kp}$ ) is the braking load opposing the motion supported by an individual, $d$ is the horizontal distance traveled by the instrumentation cylinder, $\mathrm{n}$ is the number of revolutions per minute (rpm), and $\mathrm{t}$ is the time required to move the horizontal distance. Note the difference between the concept for the prototype and the protocols of ergometry with respect to the support of the braking loads [26], in which case the dynamometer procedures are set at the maximal capacity for producing the maximal external load (kilogram or kilopound) by the dynamic or isometric action of the muscle forces. Thus, the mathematical expressions for defining these measurement methods are similar. However, for the dynamometer, the differential was assumed to be the external load that the subject must overcome in order to generate power. The external load is the force that opposes the rotational or linear motion.

Under ideal locomotion conditions, only the inertial forces acting on the motion interfere with the efficiency of the motor gesture, excluding the physiological condition of the subject [31]. The physical magnitude that defines the inertia in rotational motion is 
- Table 1 Descriptions of the components and accessories used to assemble DYNACOM.

\begin{tabular}{|c|c|c|c|}
\hline Equipment/Parts & Specifications & Picture & Value (U\$) \\
\hline $\begin{array}{l}\text { Arduino board (Atmega } \\
\text { 2586) }\end{array}$ & $\begin{array}{l}\text { Microcontroller: ATmega2560; operating voltage: } 5 \mathrm{~V} \text {; input } \\
\text { voltage (recommended): } 7-12 \mathrm{~V} \text {; input voltage (limit): } 6-20 \mathrm{~V} \text {; } \\
\text { digital I/O pins: } 54 \text { (15 provide PWM output); analog input pins: } \\
\text { 16; current per I/O pin: } 40 \mathrm{~mA} \text {; DC for } 3.3 \mathrm{~V} \text { pin: } 50 \mathrm{~mA} \text {; flash } \\
\text { memory: } 256 \mathrm{kB} \text { of which } 8 \mathrm{kB} \text { used by bootloader; SRAM: } 8 \mathrm{kB} \text {; } \\
\text { EEPROM: } 4 \mathrm{kB} \text {; clock speed: } 16 \mathrm{MHz}\end{array}$ & & 121.59 \\
\hline Rotation sensors & $\begin{array}{l}\text { Format: cylindrical; diameter: } 18 \mathrm{~mm} \text {; sensing Distance: } 5 \mathrm{~mm} \text {; } \\
\text { supply voltage: } 10-30 \mathrm{~V} \text { DC; housing: brass threaded; signaling: } \\
\text { LED; number of wires: } 2 \text {; connection: } 2 \mathrm{~m} \text { cable; maximum load } \\
\text { current: } 200 \mathrm{~mA} \text {; ambient temperature: }-10^{\circ} \mathrm{C} \text { to }+70^{\circ} \mathrm{C} \text {; degree } \\
\text { of protection: IP67; maximum operating frequency: } 500 \mathrm{~Hz} \text {; } \\
\text { hysteresis: } 5 \% \text {; standard target (steel or iron): } 18 \times 18\end{array}$ & & 60.80 \\
\hline Bike rollers & $\begin{array}{l}\text { Galvanized steel cylinders: } 3 \text { in (diameter: } 76.2 \text { mm width: } 45 \mathrm{~cm} \text { ); } \\
\text { rolling bearings; wheelbase: adaptable for } 26 \text { - and } 29 \text {-in bike rims } \\
\text { of MTB and speed bike; side steel ( } 1 / 4 \text { in } \times 11 / 4 \text { in); coated with } \\
\text { electrostatic epoxy paint }\end{array}$ & & 243.17 \\
\hline $\begin{array}{l}\text { Other components for } \\
\text { connection and logistical } \\
\text { purposes (e.g., screws, } \\
\text { bolts, wires, and connectors) }\end{array}$ & $\begin{array}{l}\text { Metal screws and lock nuts following the specifications of the } \\
\text { training rollers; security locks; metal double bearing pulleys (for } \\
\text { the calibration system); metal rings as a detection surface; } 1 \mathrm{~mm} \\
\text { thick nylon yarn to calibrate the mass support. }\end{array}$ & & 30.40 \\
\hline
\end{tabular}

the moment of inertia (MI), which is the analog of the linear motion. In a rotational motion, the $\mathrm{Ml}$ is considered to be the force opposing the motion [13]. We chose to develop a calibration mechanism that uses the $\mathrm{MI}$ in opposition to the load $(\mathrm{kg})$ according to the method described by Pupo and Ziemath [27]. An object of known mass and attached to one of the cylinders by an inextensible nylon thread is raised to a height of $0.45 \mathrm{~m}$ and then released. The dynamic force of its mass moving relative to the ground is transmitted directly to the cylinder, which turns until the moment of its stabilization. Based on the elapsed time for the entire wire to be unwound from the cylinder, the moment of dynamic inertia of the cylinder can be calculated, as shown in Eq. 2. This procedure was repeated in our laboratory with a difference of $24 \mathrm{~h}$ before data collection, and consistent results (ICC) of MI were found in both moments for both sides (right: 0.94 and left: 0.95). The value obtained in Eq. 2 can then be used in Eq. 3 to determine the propulsive power:

$\mathrm{MI}=\frac{1}{2} \mathrm{M} \cdot \mathrm{R}^{2}$

$\mathrm{PO}(\mathrm{W})=\frac{\mathrm{Ml} \cdot 0.24 \cdot \mathrm{RPM}}{1}$

where PO (Watts) denotes the gross propulsive power, $\mathrm{Ml}$ is the MI measured before the test, 0.24 is the distance in centimeters traveled by the CIN for a complete revolution, and RPM is the number of rotations performed in the given interval of time $(1 \mathrm{~s}$, multi- plied by 60 ). Although the measure is in RPM, because the measurement is performed in one-second intervals, its representation can also be provided in $\mathrm{Hz}$. Note that, during the first phase of the test, the rolling resistance values for the subject-wheelchair system were not considered because of the need to first test the applicability of the rotation sensors. This needed to be tested because the algorithms that determine the propulsive power depend on the rotation values and/or angular velocity acquired from the sensors.

- Fig. 1 shows the assembled prototype.

\section{Sample}

The subjects in this study consisted of 21 healthy male volunteers (age: $20.9 \pm 2.4 \mathrm{yr}$, body mass: $68.9 \pm 7.9 \mathrm{~kg}$, height: $174.0 \pm 7.1 \mathrm{~cm}$; BMI: $\left.22.7 \pm 2.5 \mathrm{~kg} \cdot \mathrm{m}^{2}\right)$. We chose only men to avoid changes in the circadian cycle caused by menstrual periods in females. The minimum required number of subjects was estimated a priori through a pilot study with 6 volunteers prior to the full-scale experiment. We used the G* POWER software (version 3.0.1, Faul, Germany). For this calculation, a power of 0.80 for an alpha error of 0.05 was used. The minimal sample size was estimated to be 11 subjects.

None of the recruited volunteers were wheelchair users, which complied with the guidelines set out in previous studies [22]. Nonwheelchair users were selected to increase the forces applied on the prototype and consequently produce higher levels of motion and acceleration for recording by the rotation sensors relative to those produced by people with disabilities, especially those affected by paraplegia or tetraplegia. 


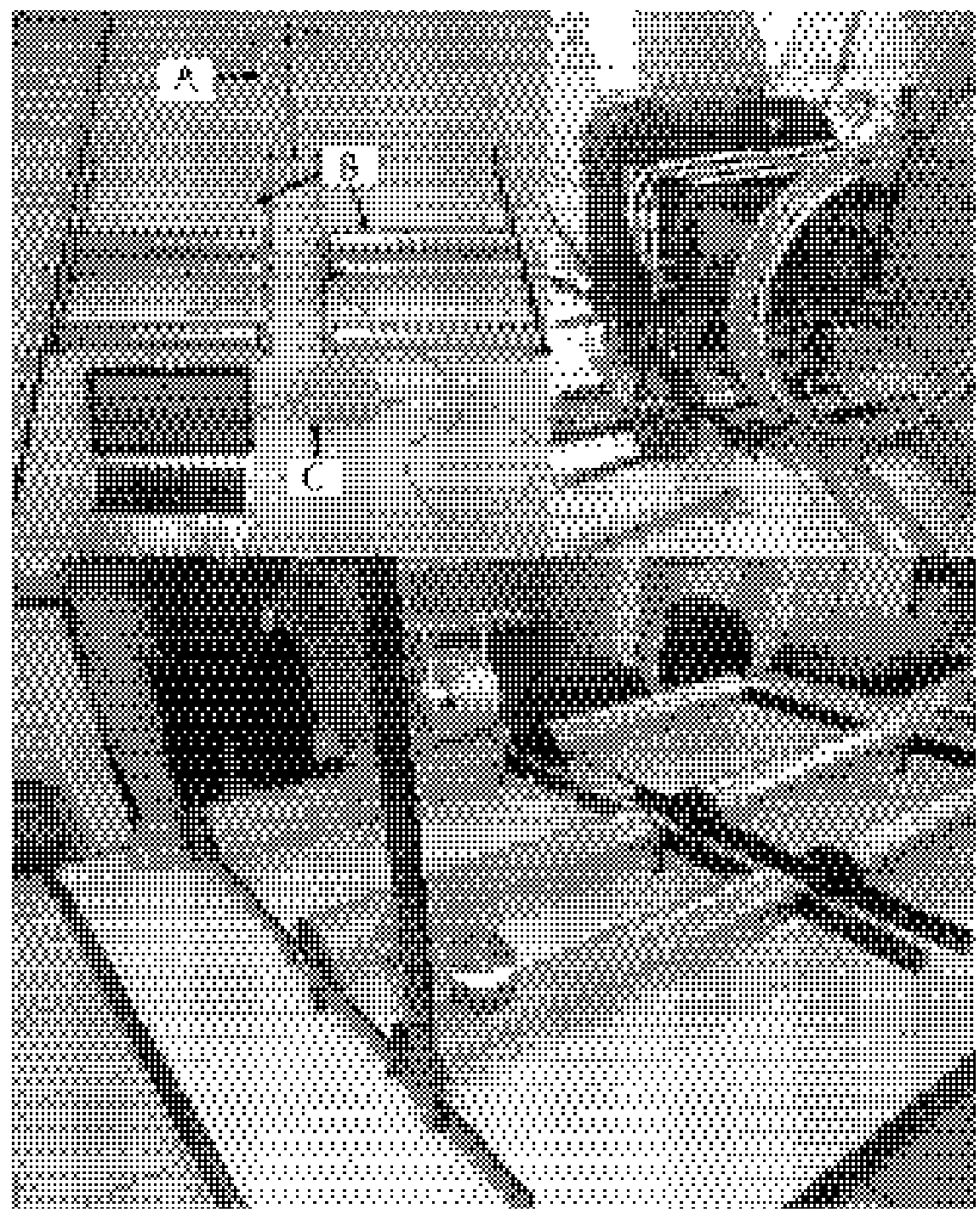

- Fig. 1 Left panel: top view of compact dynamometer with parts: a Dynamic inertia calibration system, $\mathbf{b}$ instrumentation cylinders, and $\mathbf{c}$ digitalanalog converter board. Right panel: demonstration of how the subjects were positioned for testing with DYNACOM

We considered the following criteria for inclusion in the experimental procedures: a) the subjects were not under medical supervision or specific healthcare treatment that would prevent participation in procedures with maximal stress; $b$ ) if any of the subjects reported pain or discomfort in the upper limbs, they were excluded from participation and the protocols of physical effort; $c$ ) the subjects had to be at least 18 years old; and d) they had to be male. Subjects who exhibited some impairment in the upper limbs during the data collection procedures were excluded from the sampling and analysis. After the anthropometric data were collected, each subject was positioned in a standard wheelchair with the camber axle set at $0^{\circ}$ and pre-calibrated wheels with a maximal tire pressure of $60 \mathrm{psi}$ (as indicated by manufacturers) to avoid the possible effects of unintended braking during the tests [20]. After the subjects were positioned in the DYNACOM, the wheelchair-subject set was securely fastened in the prototype with inelastic bands to avoid possible lateral or longitudinal displacements that could unbalance the subjects.

\section{Procedures}

Validation protocol for prototype with healthy subjects

The validation protocol followed the principle of the maximal stress that could be generated in the system for the acquisition and interpretation of electronic data. After all of the preparatory procedures were completed, the subjects were allowed $1 \mathrm{~min}$ to warm up and become familiar with the prototype. Each subject chose a propulsion frequency comfortable to them individually. After this period, the subjects were instructed to perform a sprint at the highest speed possible of $20 \mathrm{~s}$. During both time periods (warm-up and sprint), the selected propulsion strategy was synchronous: the subjects propelled both sides with simultaneous bilateral movements.

\section{Data collection and system of real reference}

During the validation protocol, the electric and electronic system was triggered to collect rotation readings from the right and left sensors simultaneously. Before the test began, the sensors were positioned at point zero, which was determined when the indicator LEDs were powered (connected). Before the tests, the MI was 
determined by the calibration method model, obtaining values of $0.013 \mathrm{~N} \cdot \mathrm{m}$ for both sides of the equipment. This procedure was repeated in all situations. The rotation data were immediately stored in Excel spreadsheets for further transformations in power (PO) by using the mathematical model shown in Eq. 3. The rotation values of the prototype were compared to the results produced by a reference mechanical system that simultaneously collected the actual rotation through videography (Samsung WB2000, Japan) at an image acquisition speed fixed at $240 \mathrm{fps}$; a video camera was positioned in the posterior region of the dynamometer at a distance that enabled the simultaneous recognition of both cylinders in the dynamometer (right and left). To guide analysis of the videos, reflective markers were attached to the instrumentation cylinders and in the lateral region of the dynamometer to identify the exact moment of a complete rotation. The video files were analyzed by using the open-source software VLC 2.0.1 Twoflower, Windows version (available at www.videolan.org, accessed on December 12, 2015). For temporal synchronization of the video recordings and dynamometer measurements, a mathematical procedure (Eq. 4) was used to divide the time of the videos according to the proportionality between the actual time and shooting time:

$$
\operatorname{TR}(\mathrm{s})=\left(\frac{\operatorname{TVd}(\mathrm{s})}{8}\right)
$$

where TR is the actual time (s) after correction using the image acquisition speed (240 fps), TVd is the time (s) of the original video analyzed by the researchers, and the value of 8 is the correction constant to account for the differences in signal acquisition from using the 2 procedures $(240 \div 30)$. To compare the actual (video) and experimental (prototype) values, we considered the number of rotations (ROT) and power (PO) accumulated during the first $10 \mathrm{~s}$ of the protocol based on the time range required to reach the maximal sprint speed in specific protocols for wheelchair users [6].

\section{Statistical analysis}

First, the data were analyzed in terms of their normality by using the Shapiro-Wilk test. As validation criteria, the Bland-Altman method, the intraclass correlation coefficient and the comparison test of means were defined. The latter was used if there were significant differences between DYNACOM and the reference method (high-speed cam). Next, to check the assumptions of concurrent validation, we used the Bland-Altman plotting procedures with a confidence interval of $95 \%$. The right and left sides of the prototype were compared between DYNACOM and high-speed camera. Due to the non-observance of the normal criterion for the right side of the prototype, we chose to use the Wilcoxon test for comparison of the right data, and the paired t-test for the data on the left.

In addition, as a criterion for the observation of the proportionality between the 2 methods in their absolute measures of rotation (i. e., DYNACOM and high-speed camera), the intra-class correlation coefficient (ICC) was verified by using the "one-way with fixed effects" model [34, 35]. The data were analyzed by using the GraphPad Prism version 5.0 software. In addition, the effect size was calculated for all analyses using the $G$ * POWER ${ }^{\circledR}$ software and according to Hopkins [14], as verified in previous studies [4]. For all of the analyzed conditions, a significant P-value of less than or equal to $5 \%(P \leq .05)$ was established.

\section{Results}

- Table 2 lists the individual power output values obtained with both methods: high-speed camera and DYNACOM.

- Fig. 2 shows the Bland-Altman plots, comparing DYNACOM and the reference method (high-speed cam). All values were within the limits of accordance established by the method with a confidence interval of $95 \%$ for both sides of the prototype. We emphasize that only the right side of the equipment observed the lower and upper limits of agreement, considering that the left side of the equipment presented identical measures of rotation and power in comparison with the method using a high-speed cam (actual rotation). The bias values of both the variables for the right side (rotation: .14 and power: .00) were considered insignificant based on the hypothesis test for reference samples $(P>0.05)$.

In terms of the concurrent validity, the values obtained from the intra-class correlation coefficient were excellent (i. e., higher than 0.9 ), in agreement with the recommendations in [1]. Significant values were observed for both the rotation data and power on both sides of the prototype (right and left $p<0.00$ ). Similarly, no statistically significant differences were observed when the number of rotations and the power were analyzed. Both were measured after $10 \mathrm{~s}$ of the protocol for sprinting at maximal speed, as shown in

Fig. 3 .

- Table 2 Individual values of the power output acquired with DYNACOM.

\begin{tabular}{|c|c|c|c|c|}
\hline \multirow[t]{2}{*}{ Subjects } & \multicolumn{2}{|c|}{ Right side (power, W) } & \multicolumn{2}{|c|}{ Left side (power, $\mathrm{W}$ ) } \\
\hline & $\begin{array}{l}\text { High-speed } \\
\text { camera }\end{array}$ & DYNACOM & $\begin{array}{l}\text { High-speed } \\
\text { camera }\end{array}$ & DYNACOM \\
\hline 1 & 0.15 & 0.15 & 0.20 & 0.20 \\
\hline 2 & 0.17 & 0.17 & 0.24 & 0.24 \\
\hline 3 & 0.13 & 0.13 & 0.18 & 0.18 \\
\hline 4 & 0.19 & 0.18 & 0.23 & 0.23 \\
\hline 5 & 0.12 & 0.12 & 0.20 & 0.20 \\
\hline 6 & 0.16 & 0.16 & 0.19 & 0.19 \\
\hline 7 & 0.16 & 0.16 & 0.19 & 0.19 \\
\hline 8 & 0.13 & 0.13 & 0.13 & 0.13 \\
\hline 9 & 0.16 & 0.16 & 0.17 & 0.17 \\
\hline 10 & 0.21 & 0.21 & 0.16 & 0.16 \\
\hline 11 & 0.19 & 0.19 & 0.23 & 0.23 \\
\hline 12 & 0.16 & 0.16 & 0.19 & 0.19 \\
\hline 13 & 0.28 & 0.28 & 0.25 & 0.25 \\
\hline 14 & 0.15 & 0.15 & 0.15 & 0.15 \\
\hline 15 & 0.18 & 0.18 & 0.20 & 0.20 \\
\hline 16 & 0.19 & 0.19 & 0.22 & 0.22 \\
\hline 17 & 0.16 & 0.16 & 0.20 & 0.20 \\
\hline 18 & 0.19 & 0.19 & 0.22 & 0.22 \\
\hline 19 & 0.20 & 0.20 & 0.21 & 0.21 \\
\hline 20 & 0.17 & 0.17 & 0.21 & 0.21 \\
\hline 21 & 0.21 & 0.21 & 0.26 & 0.26 \\
\hline Average & 0.17 & 0.17 & 0.20 & 0.20 \\
\hline $\begin{array}{l}\text { Standard } \\
\text { deviation }\end{array}$ & 0.04 & 0.03 & 0.03 & 0.03 \\
\hline
\end{tabular}

Effect size for the differences between the number of revolutions, real (high-speed) and measured (prototype) $=1.11$ 

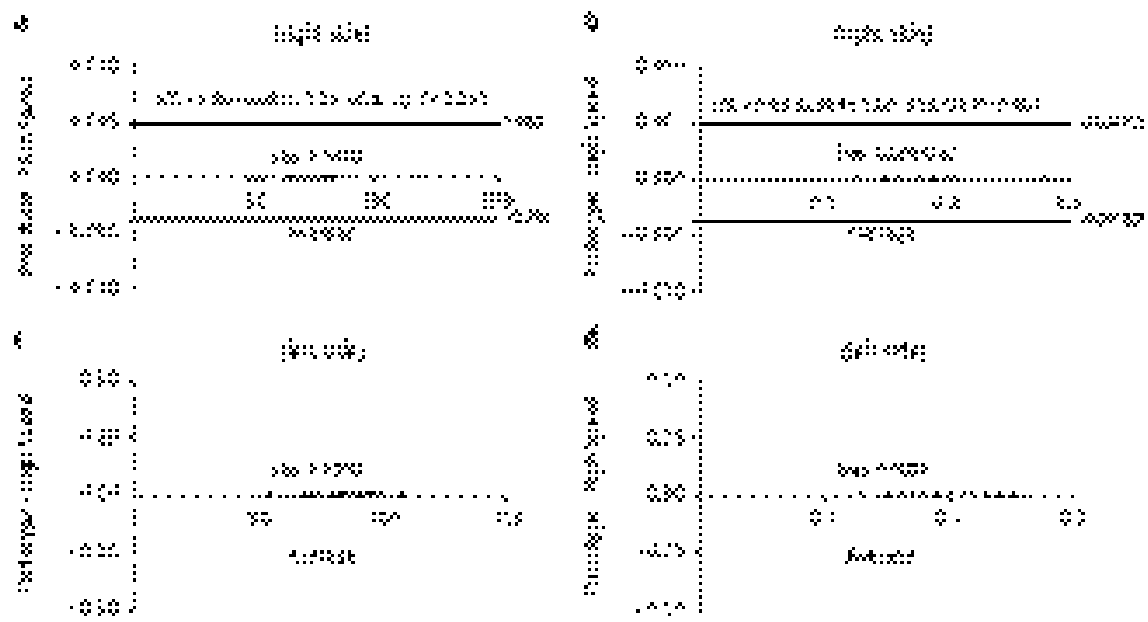

- Fig. 2 Bland-Altman plots of cumulative rotation and power for the $10 \mathrm{~s}$ sprint on the right and left sides of the prototype: cumulative rotation and power output on the right side (panels $\mathbf{a}$ and $\mathbf{b}$ ); cumulative rotation and power output on the left side (panels $\mathbf{c}$ and $\mathbf{d}$ ). $\mathbf{N}=21 ;{ }^{*} p<0.00$; Highspeed: high-speed camera; Prototype: DYNACOM.

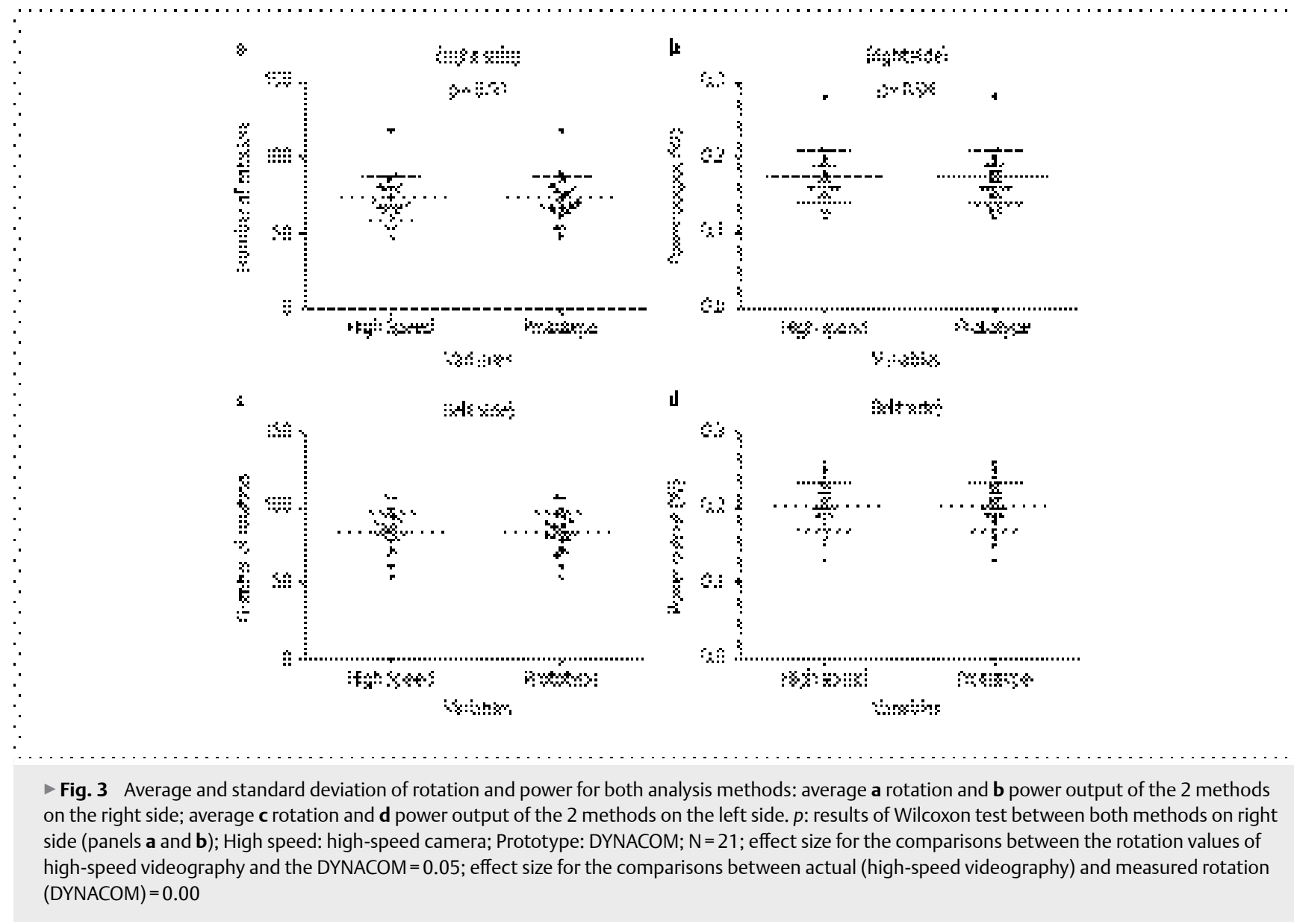




\section{Discussion}

The aim of the present study was to develop and validate new equipment for measuring and evaluating the propulsive power of wheelchair users. One aspect of particular interest in the scientific literature on performance evaluation is related to the validity of the scientific methods and techniques available in several areas. When designing and testing protocols for the functional assessment of disabled subjects, the validity of the data is a key component that provides professionals with accurate information about phenomena related to ergonomics and movement efficiency. Thus, knowing the actual scientific conditions associated with specific equipment enables evaluators to make safe decisions without interpreting measurements above or below the actual values.

In the present study, the Bland-Altman method [7, 25] was chosen as the main tool for verifying the validity and reliability of the information. The previously mentioned statistical process allowed simultaneous analyses of both methods with different definitions for the limits of accordance. At a confidence interval of $95 \%$, the data for both motion sensors (left and right) were within the accordance limits determined by the method. In addition, for the sensor on the right side, the bias did not show a statistically significant difference in the analysis of the hypothesis test $(P>0.05)$.

Aspects of particular interest in this field are the factors that may be influencing rolling resistance. In our study, this variable was not analyzed because it is an ancillary aspect of manual propulsion capacity evaluation procedures. However, studies have shown that rolling resistance is an inherent aspect of the subject and wheelchair assembly [30,31].

Recently, Terran and Ueda [36] observed a proportional relationship between velocity, acceleration, and rolling resistance, suggesting the need to control the factors that may influence this condition, while attenuating the negative influence and potentiating the positive contributions of this aspect to locomotion in wheelchairs.

For one subject, the right-side sensor presented rotation values that were less than the actual values with differences of up to 2 rotations. Some hypotheses can be given to explain this phenomenon. First, this was a prototype that included a weight support system (subject + wheelchair) and an electronic system for measuring and verifying the power. Specifications such as the console for accessing the dynamometer and the accommodation platform for the front wheels of the chair still need to be modeled based on the individual needs of each subject, for example, by adding ramps to smooth mobility cylinders. In the experimental sessions, this support was provided with adapted platforms; this factor undoubtedly made the balance and movement of the subjects during the tests slightly more difficult.

Another detail of particular importance concerns the connectors between the inductive motion sensors and side stems of the equipment. Based on the physical and mechanical specifications of these sensors, the minimum distance for activation and detection was $0.01 \mathrm{~m}$. Considering this small distance of activation for the sensor operation, vibrations produced from the manual propulsion by the subject may have been responsible for the loss of a small number of signals. As a limitation, this research lacked a console that would allow lower vibrations during the stress tests. For the next testing of the prototype, supporting materials that lower the influence of vi- bration and unwanted movements during the stress testing will be used. In tests with wheelchair users, this would usually be a necessary solution to avoid greater measurement errors.

With regard to the power ( $\triangleright$ Table 1 ), we were able to detect smaller values compared with other studies $[8,9,12]$. This was due to the differences in verification systems for the propulsive and power forces. The models suggested in $[6,8]$ used torque sensors mounted directly on the axes of the ergometer in addition to the increments of the electromagnetic brake systems. This added to the weight of the subject and inertia provided by the cylindrical framework, which generated higher values of PO compared to the prototype validated in this research. With regard to the differences between the proposed and existing prototypes to date, however, the main characteristic of the latter is the imposition of a load that breaks the propulsive movement, which consequently requires adaptation by the subject being assessed during the test. The equipment described in this paper works in reverse: it measures the largest power generation capacity from the standardized effort and possible changes that may be caused by the user-wheelchair ratio.

Other models of dynamometers, such as those described in $[5,30]$, have costly and complex calibration methods that use the load cell instrumentation to verify the power involved in the rotational movement and tachogenerators that measure the movement and power of the cylinders. The mechanism developed in this work is based on the transmission of potential and kinetic energy from the free fall of a known mass [27]. This makes the assessment process faster and allows it to be performed repeatedly and with different evaluators without the need for extensive training. Note that the values of the MI found in this study differ from those proposed in previous studies [5].

The present study was limited to the evaluation of non-wheelchair users. This procedure is in accordance with previous studies [22] that used non-disabled subjects because they are less susceptible to predetermined standards of manual propulsion, which would require significant changes in the handling of preferred strategies for each subject. Another consideration was the need for ideal conditions for shooting at high speed, which included the requirement of sufficient lighting to correct for possible decreases in the image resolution quality. These conditions could only be achieved with a significant number of subjects in the laboratory. Thus, excellent validation results were observed based on the previously plotted parameters (ICC and Bland-Altman plots). Future research should focus on determining the most suitable protocols for each disability and the level of physical activity or sport modality, as well as adapting the evaluation of the propulsive power to the required effort and individual efficiency of distinct groups.

Limitations of the present study include the use of an exclusively male sample and the non-assessment of rolling resistance. As practical implications of our findings, we can determine the possibility of evaluating wheelchair users at their own training and rehabilitation sites because of the ease of transport of the equipment. However, experienced kinesiologists are required to handle the prototype for ensuring its proper use because other software features are required for data collection and recording. A simple interface is being developed at the moment to facilitate its use by other professionals who work in the area of rehabilitation or physical conditioning. 
Other studies need to be conducted to consider the rolling resistance values for the movement during wheelchair propulsion $[20,21,31]$. These variables may better reflect functional information regarding the propulsion capacity and contribute to research on the maneuverability, accessibility, and ergonomics of wheelchairs, whether for activities of daily living or sports.

\section{Conclusion}

The proposed prototype demonstrated the required scientific reliability for evaluation of wheelchairs used by athletes or sedentary people. We therefore propose further studies be undertaken to verify the best effort protocols with regard to the type of wheelchair, degree of disability, and experience with using the wheelchair in order to identify the most efficient conditions for physical and ergonomic performance.

\section{Acknowledgements}

Acknowledgements: We thank students Mirella Cabral, Felipe Freitas, Humberto Morales and Juliana Brito for their help with the construction of the prototype phase, in addition to Professor Renato Moraes for giving the premises of the Education Centre Distance (University of Pernambuco) for this phase of the research. Financial funds: The materials used in the study were provided by the University of Pernambuco, and the financial support was kindly provided by the Brazilian Government through the Ministry of Sports.

\section{Conflict of Interest}

The authors declare that they have no conflict of interest.

\section{References}

[1] Atkinson G, Nevill AM. Statistical methods for assessing measurement error (reliability) in variables relevant to sports medicine. Sports Med 1998; 26: 217-238

[2] Cooper RA, Robertson RN, VanSickle DP, Boninger ML, Shimada SD. Methods for determining three-dimensional wheelchair pushrim forces and moments: a technical note. J Rehabil Res Dev 1997; 34: 162-170

[3] Cooper RA. SMARTWheel: From concept to clinical practice. Prosthet Orthot Int 2009; 33: 198-209

[4] Dantas JL, Pereira G, Nakamura FY. Five-kilometers time trial: preliminary validation of a short test for cycling performance evaluation. Asian J Sports Med 2015; 6: e23802

[5] DiGiovine CP, Cooper RA, Boninger ML. Dynamic calibration of a wheelchair dynamometer. J Rehabil Res Dev 2001; 38: 41-56

[6] Devillard X, Calmels P, Sauvignet B, Belli A, Denis C, Simard C, Gautheron V. Validation of a new ergometer adapted to all types of manual wheelchair. Eur J Appl. Physiol 2001; 85: 479-485

[7] Dewitte K, Fierens C, Stöckl D, Thienpont LM. Application of the Bland-Altman plot for interpretation of method-comparison studies: a critical investigation of its practice. Clin Chemistry 2002; 48: 799-801

[8] Faupin A, Campillo P, Weissland T, Gorce P, Thevenon A. The effects of rear-wheel camber on the mechanical parameters produced during the wheelchair sprinting of handibasketball athletes. J Rehabil Res Dev 2004; 41: 421-428
[9] Faupin A, Gorce P, Thevenon A. A wheelchair ergometer adaptable to the rear-wheel camber. Int J Ind Ergon 2008; 38: 601-607

[10] Finley MA, Rasch EK, Keyser RE, Rodgers MM. The biomechanics of wheelchair propulsion in individuals with and without upper-limb impairment. J Rehabil Res Dev 2004; 41: 385-394

[11] Gil-Agudo A, Del Ama-Espinosa A, Pérez-Rizo E, Pérez-Nombela S, Rodríguez-Rodríguez LP. Upper limb joint kinetics during manual wheelchair propulsion in patients with different levels of spinal cord injury. J Biomech 2010; 43: 2508-2515

[12] Goosey-Tolfrey V, Castle P, Webborn N. Aerobic capacity and peak power output of elite quadriplegic games players. $\mathrm{Br}$ J Sports Med 2006; 40: 684-687

[13] Hall S], Taranto G. Biomecânica básica. Rio de Janeiro: Guanabara Koogan; 2000

[14] Hopkins WG. Measures of reliability in sports medicine and science. Sports Med 2000; 30: 1-15

[15] Hu X, Blemker SS. Musculoskeletal simulation can help explain selective muscle degeneration in Duchenne muscular dystrophy. Muscle Nerve 2015; 52: 174-182

[16] Hughes TC, Coleman T, Hellar DB, Vega LC, Patterson ES. Web application dashboards from intelligence analysis to health care: applying the open-source OZONE widget framework to health IT. Proc Int Symp Hum Factors Ergon Healthc 2014; 3: 186-190

[17] Jaspers E, Monari D, Molenaers G, Feys H, Desloovere K. ULEMA-Upper limb evaluation in movement analysis: Open-source custom made MATLAB based software. Gait Posture 2014; 39: S76-S77

[18] Keyser RE, Rasch EK, Finley M, Rodgers MM. Improved upper-body endurance following a 12-week home exercise program for manual wheelchair users. J Rehabil Res Dev 2003; 40: 01-510

[19] Kwarciak AM, Turner JT, Guo L, Richter WM. The effects of four different stroke patterns on manual wheelchair propulsion and upper limb muscle strain. Disabil Rehabil Assist Technol 2012; 7: 459-463

[20] Kwarciak AM, Yarossi M, Ramanujam A, Dyson-Hudson TA, Sisto SA. Evaluation of wheelchair tire rolling resistance using dynamometerbased coast-down tests. J Rehabil Res Dev 2009; 46: 931-938

[21] Lemaire ED, Lamontagne M, Barclay HW, John T, Martel G. A technique for the determination of center of gravity and rolling resistance for tilt-seat wheelchairs. J Rehabil Res Dev 1991; 28: 51-58

[22] Lenton JP, van der Woude L, Fowler N, Goosey-Tolfrey V. Effects of arm frequency during synchronous and asynchronous wheelchair propulsion on efficiency. Int J Sports Med 2009; 30: 233-239

[23] Motl RW, Fernhall B. Accurate prediction of cardiorespiratory fitness using cycle ergometry in minimally disabled persons with relapsingremitting multiple sclerosis. Arch Phys Med Rehabil 2012; 93: 490-495

[24] Morrow MM, Kaufman KR, An KN. Shoulder model validation and joint contact forces during wheelchair activities. J Biomech 2010; 43: 2487-2492

[25] Myles PS, Cui Jl. Using the Bland-Altman method to measure agreement with repeated measures. Br J Anaesth 2007; 99: 309-311

[26] Niesing R, Eijskoot F, Kranse R, den Ouden AH, Storm J, Veeger HE, Snijders CJ. Computer-controlled wheelchair ergometer. Med Biol Eng Comput 1990; 28: 329-338

[27] Pupo HC, Ziemath EC. Determinação do momento de inércia de um volante usando um faiscador. Cad Braz Ens Fis 2004; 21: 69-75

[28] Rice I, Gagnon D, Gallagher J, Boninger M. Hand rim wheelchair propulsion training using biomechanical real-time visual feedback based on motor learning theory principles. J Spinal Cord Med 2010; 33: 33

[29] Schmidt A, Huonker M, Stober P, Barturen JM, Schmidt-Trucksäss A, Dürr $\mathrm{H}$, Keul J. Physical performance and cardiovascular and metabolic adaptation of elite female wheelchair basketball players in wheelchair ergometry and in competition. Am J Phys Med Rehabil 1998; 77: 527-533 
[30] Shimada SD, Cooper RA, Lawrence B, Robertson RN. (1995, September). Computer controlled wheelchair dynamometer. In Engineering in Medicine and Biology Society. IEEE 17th Annual Conference 1995; 2: $1177-1178$

[31] Theisen D, Francaux M, Fayt A, Sturbois X. A new procedure to determine external power output during handrim wheelchair propulsion on a roller ergometer: A reliability study. Int J Sports Med 1996; 17: 564-571

[32] Van der Scheer JW, de Groot S, Postema K, Veeger DH, van der Woude $\mathrm{LH}$. Design of a randomized-controlled trial on low-intensity aerobic wheelchair exercise for inactive persons with chronic spinal cord injury. Disabil Rehabil 2013; 35: 1119-1126
[33] van der Woude LH, Veeger HE, Dallmeijer A], Janssen TW, Rozendaal LA. Biomechanics and physiology in active manual wheelchair propulsion. Med Eng Phys 2001; 23: 713-733

[34] Vincent W, Weir J. Statistics in kinesiology. Human Kinetics, 4a ED 2000

[35] Weir JP. Quantifying test-retest reliability using the intraclass correlation coefficient and the SEM. J Strength Cond Res 2005; 19: 231-240

[36] Teran E, Ueda J. Influence of rolling resistance on manual wheelchair dynamics and mechanical efficiency. Int J Intell Robot Appl 2016; 1-19

[37] Harriss DJ, Atkinson G. Ethical standards in sports and exercise science research: 2016 update. Int J Sports Med 2015; 36: 1121-1124 